\title{
Blood Flow at Arterial Branches: Complexities to Resolve for the Angioplasty Suite
}

\author{
P.D. Richardson ${ }^{1}$, I.V. Pivkin ${ }^{2}$, G.E. Karniadakis ${ }^{2}$, and D.H. Laidlaw ${ }^{3}$ \\ ${ }^{1}$ Division of Engineering \\ ${ }^{2}$ Division of Applied Mathematics \\ ${ }^{3}$ Department of Computer Science \\ Brown University, Providence, RI 02912, USA
}

\begin{abstract}
This paper presents a case of interdisciplinary collaboration in building and using a set of tools to compute the flows in a branched artery, to compare them with prior physical flow visualization, and to interpret them with further users in mind. The geometry was taken for a typical epicardial coronary artery with a side branch. The incompressible Navier-Stokes equations were solved with the hybrid spectral/hp element solver Nektar. Some simulations were visualized in the CAVE, an immersive $3 \mathrm{D}$ stereo display environment, and selected features are described and interpreted.
\end{abstract}

\section{Introduction}

Two papers by Colin Caro and his colleagues in the 1970s 1, 2, led to a large increase of interest in the possible role of fluid dynamics in the development of atherosclerosis in arteries, and the consequent impact of this focal and local vessel disease. This disease evolves over time to be critical in chronic conditions such as claudication (from ischemic effects in the legs), transient ischemic effects in the brain (often followed within 6 months or so by thrombotic strokes), and myocardial infarctions due to obstruction in coronary arteries. The local pathology of atherosclerotic plaques is widely found in many arterial locations, in vessel sizes ranging from the aorta down to arteries a tenth or so of the aortic diameter [3, 4. A pathological finding, that prompted Caro et al to suggest a role of local variations in blood flow patterns, is that the distribution of such atherosclerotic lesions on blood vessel walls is not random, but has higher frequency of incidence closely downstream of arterial branches [1,2. Clinically the acute and locally-focussed treatment of coronary artery disease is undertaken widely in the angioplasty suite. Cylindrical balloons are introduced to coronary arteries and inflated to open up stenotic sections. Often a physical prop, a stent, is inserted by balloon to hold open a section that had been stenosed (bottlenecked) by the disease. More recently, a catheter-based device has been approved for using a fine wire with a coiled tip to be screwed into a thrombus in the carotid artery and withdraw it, to clear a passage for blood flow there.

Despite many advances made in understanding cell structure and function in the past 30 years, especially regarding transmembrane channels, receptors and 
the cytoskeleton, as well as in arterial vessel mechanics and function, including the concept of the vulnerable plaque, on the one hand, and many flow computation and visualization studies in branched and curved tubes on the other hand, no clear closure to the question of why plaques develop spatially non-randomly on arterial walls has been achieved. Even in the flow computations and visualizations, little progress has been made in investigating how well the observations can be explained by combination of prior knowledge of specific flow situations, examined for their breadth by comparison with specific examples. In this paper some selected examinations of the flow in a circular tube in the vicinity of a side branch are described and discussed, first from using regular flow visualization with dye streams and secondly using post-processing of computed flows; the latter embrace tube geometric modifications not achieved in regular physical flow visualization.

\section{Physical Flow Visualization for Branched Tubes}

Branched tubes were fabricated by machining - drilling, reaming, and chloroformpolishing - of 3mm-diameter straight holes in clear PMMA flat-sided blocks, with $1.5 \mathrm{~mm}$-diameter side-branches introduced with axes intersecting those of the primary holes and, in different blocks, having the side branches with their axes at 45 or 90 degrees from the downstream direction of the main $3 \mathrm{~mm}$ holes. Directly upstream of the blocks, and with their central axes and internal diameter matching the $3 \mathrm{~mm}$ hole in the block, there were two short tube sections each of which had one small hole permitting a dye stream to be added to the flow, and capable of being rotated so the dye streams from each could be admitted at a selected and measured circumferential angle relative to that of the axis of the side branch downstream. Careful fitting, including use of O-ring seals, assured that the angle of each dye stream could be altered as desired without leaks occuring. Photographs of flow patterns as shown by dye flows were taken with a $35 \mathrm{~mm}$ Pentax camera having a macro lens.

A typical steady-flow visualization is shown in Figure1. The flow, passing from left to right, comes very close to the tube junction before showing any alteration from straight laminar flow. In the region where the flow divides the dye streams broaden; because the dye streams have a finite diameter as they approach, this makes clear that the flow develops a different direction close to the wall than at small distances radially inwards. Broadly speaking, one expects the flow close to the wall, having relatively little momentum, to be more sensitive to the local pressure gradient than portions of the flow closer to the center of the stream. The pressure gradient is expected to have components both across the main tube and along it. The transverse component is due to distribution of the reaction force associated with providing the momentum for the portion of the flow going down the side branch. The longitudinal component of pressure gradient is associated with the divergence of streamlines, and the corresponding deceleration of the portion of the fluid stream entering the exit section of the $3 \mathrm{~mm}$-diameter tube. This divergence reminds one of a classic solution in fluid dynamics, that of steady 


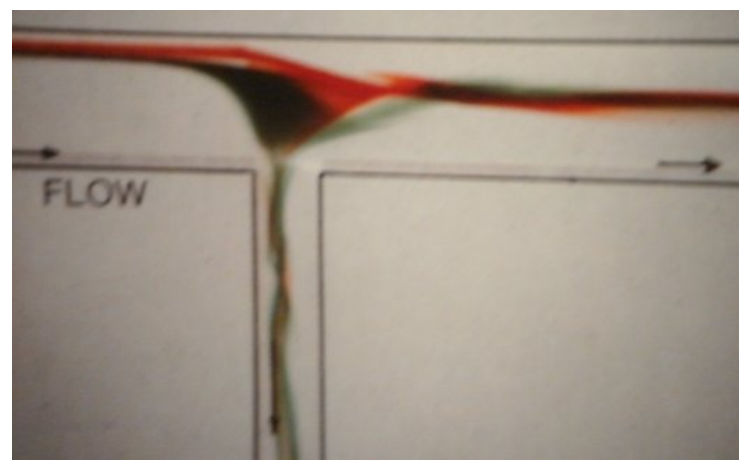

Fig. 1. Steady flow in a tube with a side branch. Visualization illustrating a case with flow separation opposite a side branch.

flow in a conical, diverging tube, and it is interesting that this comparison leads to a prediction of flow conditions - basically a range of values of the side-branch flow rate divided by the downstream main-tube flow rate, as a function of the Reynolds number of the flow upstream of the branch - for which flow separation on the side of the main-tube wall opposite the side-branch, and continuing some distance downstream, is observed. This was reported first as a correlation 5] in 1985 and subsequently with an outline of the analysis [6] at a conference of flows in large blood-vessels held in 1989. Our own computations have confirmed this behavior. Associated with a separated flow there is a significant change in the shear stress in the flow at the wall, and the prospective impact of this on lipid transport between a blood stream and a vessel wall in this region - which happens to be where atherosclerotic plaques are found more frequently - was discussed by Caro et al [1,2].

When the inflow was made pulsatile, the dividing line (between having flow separation or not on the portion of the wall opposite and downstream of the opening into the side branch) broadened into a wedge shape: above the upper line of the wedge the flow was separated there throughout a pulsation, in between the two lines the flow was episodically separated/attached through a pulsation, and below the lower line the flow did not separate at any time during the cycle of a pulsation. The lines came together when the inflow Reynolds number at the upstream portion of the branched tube approached 1,000.

Crossing to the side where the side branch entrance is, the sharp transition of the wall for flow coming along the upstream wall to the side branch leads to a flow separation in the corresponding portion of the entrance section of the side branch over a wider range of flow conditions. This was visible with regular flow visualization, as illustrated in Figures 2(a) and 2(b), where a branch at 45 degrees was examined - this made it easier to photograph the flow from what is the lower-left position in Figure 2(a) to provide Figure 2(b) than would have been the case with a 90-degree branch. Two dyestreams of different colors were applied upstream, and the dye tracks show the dye swerving to enter the side branch in the mid-position across it entrance mouth as seen in Figures 2 (a) and 2 (b), the 

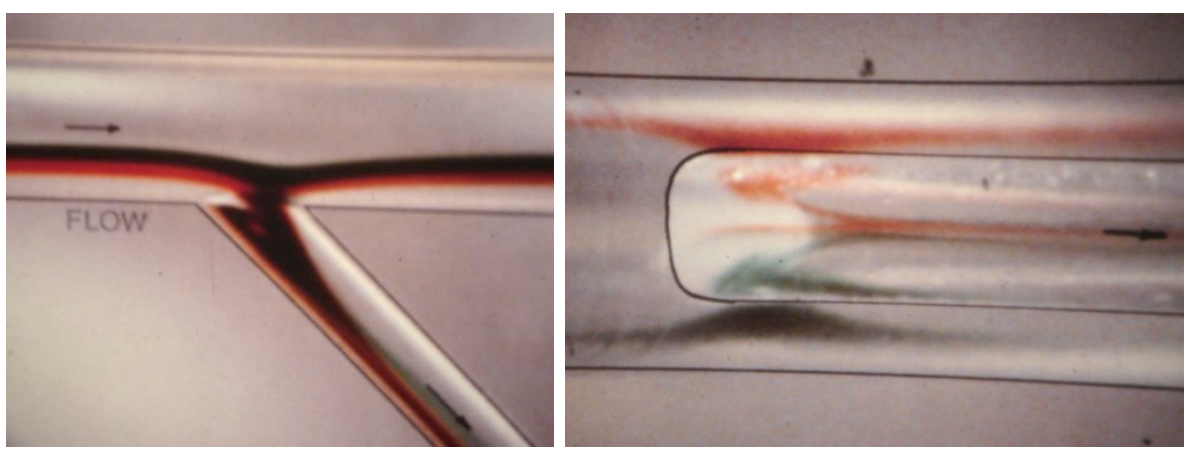

Fig. 2. Steady flow in a tube with a side branch: flow separation in the side branch. (a) Visualization from a side. (b) Visualization from under side branch.

dyestreams approach each other on the underside of the side branch roughly two diameters downstream of the entrance and then each divides, some moving retrograde by the wall and towards the entrance and some continuing downstream. The retrograde flow, on coming very close to the entrance, passes under the flow which has separated at the upstream edge and is carried downstream in the side branch. The length of this region with reversed flow was observed to increase down the side branch as the Reynolds number of the flow in the side branch increased.

\section{Visualization of Branched-Tube Flows Derived from Computations}

We have made computations for flows, steady and unsteady in branched tubes similar to, and also modified from, those use in the earlier experiments. One modification was to provide curvature to the axes of the tube section, to study more some details of the flow that would be expected in epicardial coronary arteries, which ride in grooves on the curved external suface of the heart. It has been known for several decades following the work of Dean [7] that a secondary motion is found in steady flow in curved tubes.

We thought it would be interesting to examine vortex lines in the flow, and developed some by putting seed points in the flow, computing the local vorticity, marching a small distance in the direction in which it pointed, computing the local vorticity at that point, revising the direction in which to march to the next point, and continued for a few thousand small steps before stopping arbitrarily. If a seed point fell at the edge of the flow field and marching produced a straight line, this was terminated much sooner as an artefact. (In any case, because of the arbitrariness of seed locations and of terminations there would be some lines that appeared to start and finish in the flow, another minor artefact). To visualize the lines they were each sheathed visually with a thin uniform tube, and then displayed as in Figure 3(a). As expected, the central core of the flow showed many similar lines in tight coilings traced in each such sequence, with those in 

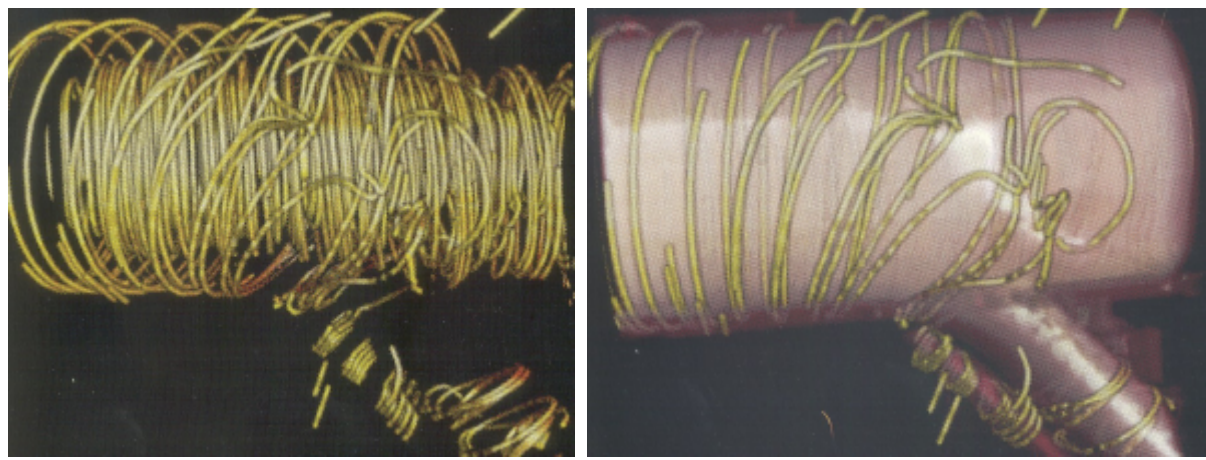

Fig. 3. Steady flow in a tube with a side branch: sample vortex lines from computed flows. (a) Starter tracking points seeded throughout flows in tube. (b) Sheath, of 0.1 mean velocity magnitude surface, inserted to make only vortex lines close to wall distinct.

the outer 10 per cent or so of the radius being inclined differently. To reduce the visual distraction from the inner coiling, an opaque sheath (with some specular reflectivity) was created where the magnitude of the local velocity was 0.1 of the mean velocity in the inflow. A typical resulting image, again close to the side branch, is shown in Figure 3(b). A feature observed here is the formation of 'hairpins' in a few of the vortex lines. These are evocative of similar hairpins that form in laminar boundary layers as part of the process of transition to turbulence, and for any given Reynolds number the likelihood of their being stimulated is increased when there is an 'adverse' pressure gradient, as occurs in this region. This hairpin shape was not observed to evolve downstream, where the pressure gradient stopped being 'adverse', and in this case no early transition to turbulence was indicated. However, it was very interesting to see this, which physical flow visualization was extremely unlikely to catch. It was present in this case only on the side where the vessel wall had negative Gaussian curvature, it was looked for on the opposite side (not shown) but was not present there.

The presence of the reverse flow region at the upstream side of the side branch, under a locally separated flow entering the side branch from the upstream supply, was made visible by retaining the sheathing of regions by local magnitudes of 0.1 mean velocity. Use of the magnitude ignores the local direction of flow. Figure 4 shows a close-up of the region close to the entrance in to a 45-degree branch, and in this Figure the position of the tube walls has not been ghosted in. Visible in this Figure is an object like a finless fish. Because the flow velocity changes direction between its upper surface and the main flow coming into the side branch, there is a seeming gap between them where the local velocity is changing direction and is therefore slow enough to be at less than 0.1 of the entering mean flow. Similarly, to the left of the underside of the 'finless fish' there is a region where the local velocity is less than 0.1 , and the location of the tube wall is separated from the visible surface of the 'fish'. With this understanding, the similarity of the phenomenon of a reversed flow region displayed in the images 


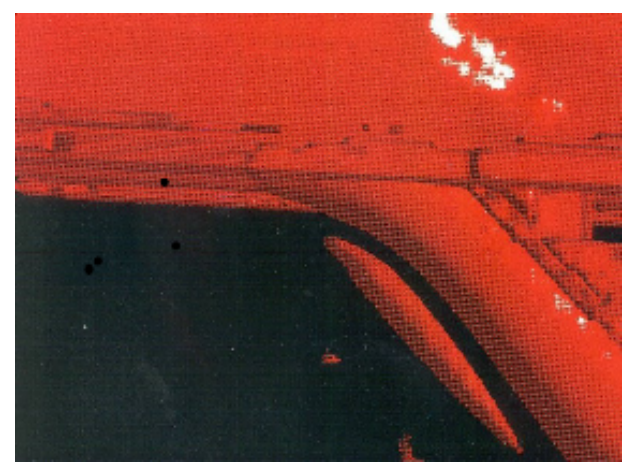

Fig. 4. Steady flow in a tube with a side branch: surfaces of 0.1 mean velocity magnitude surface shown. Detail near entry to side branch, with 'finless fish' showing part of reverse flow region.

in Figures 2(a) and 2(b) and Figure 4 can be appreciated, in the region just downstream of the lip of the side branch. This is undoubtedly of relevance to tubing geometries in manufactured equipment. Arteries are somewhat rounded locally in this region, and it is likely that the Reynolds number of the approaching flow has to be large enough, or accelerated rapidly enough, for a similar flow separation over a reverse flow region to be present there.

Another feature of the flow in regions affected by a side-branch in tubing geometry is secondary motion. Dean-type secondary motions in curved tubes are already well known, and are driven basically by an imbalance of centrifugal forces in them. The secondary motion involves flow across the diameter of the tube, in the plane of its curvature, and running from the tube wall at the inside of the curve to the outside of the curve, with a compensating flow (to satisfy continuity requirements) close to the wall surfaces that bracket the plane of the curvature. Thus the flow moves around in a "D" shape at each side of the plane of the tube curvature. The secondary flow requires a finite arc of curvature to develop into its full strength: however, this is a fraction of a radian. Even with straight tube sections, the diversion of part of a straight-flowing stream through an angle into a side branch involves a finite period where there can be an imbalance of centrifugal forces across curved streamlines, and a turn of the order of a radian. Flow visualizations (not shown here) suggest that a pair of counter-rotating secondary-flow cores are present for some diameters downstream in a side-branch tube. There may be some in the downstream continuation of the main tube too, especially if there is flow separation opposite the side branch, as the separation region may be occupied by a horse-shoe vortex. Dean-type secondary motions can persist even in the presence of some pulsatility in the flow, and indeed there is some evidence that pulsatility added to a Dean-type secondary motion may increase the mean mass or heat transfer coefficient in a curved tube [8] compared with a straight tube having the same flow through it. Such an increase in mean transfer rates likely involves even larger variations in local transfer coefficients at different circumferential locations. Because the development of atherosclerotic lesions may be 

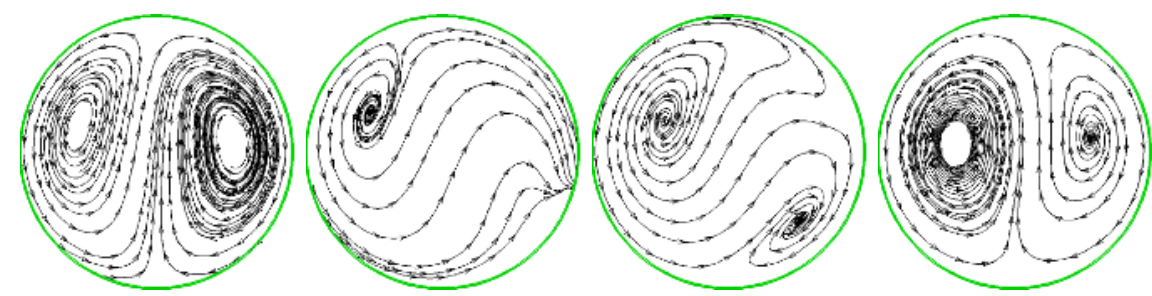

Fig. 5. Secondary flow patterns in main tube from our computations, successively (i) at one tube diameter upstream of intersection of tubing axes (ii) at intersection point of tubing axes (iii) one diameter downstream (iv) three diameters downstream, together showing range of slewing-round of the secondary flow by the flow exiting down the side branch.

affected by local convective lipid transport coefficients, this needs to be examined in greater detail. With space limitations here, we demonstrate one feature relating to secondary flows and a side branch.

In Figure 5 we show a sequence of patterns of secondary flow in the main tube flow, starting one tube-diameter upstream of the point where the axis of the side branch meets the axis of the main tube; at this position the secondary flow is almost identical to that in a curved tube without a side branch. The next secondary flow pattern is at the meeting point of the vessel axes; to the right on this pattern there are streamlines converging towards 3 o'clock, corresponding to contribution to the flow into the side branch, and the vorticity core that had been on the left in the previous flow slewed round roughly 45 degrees clockwise. One diameter further downstream in the main branch the slewing of the vortex structures persists, but with the outflow to the side branch now passed the second vortex core begins to reorganize. By three diameters downstream of the meeting-point of the vessel axes the secondary streamlines are close to normal again. Because this variation of the secondary flow - uniquely made visible by examining the computed flows - will be accompanied by local variations of the mass transport rate at the wall in a way unlikely to be symmetric about the midplane through the parent artery and its side branch, some resolution of the possible role of local transport of lipids may be determined by examining pathological specimens for helical slewing of the atheroma that develop over the range in a parent vessel (such as the left anterior descending coronary artery), downstream of a side branch, in which the secondary flow itself is slewed. Additionally, observations can be made from use of intravascular ultrasound in the angioplasty suite to similar purpose. With pulsatile flows, as was well demonstrable by viewing streamline patterns close to the wall in a CAVE, the secondary flow is strongly sensitive to the accelerating phase in its response.

\section{Discussion and Conclusions}

Prior experimental work, of which some previously unpublished flow visualization (by the senior author with J. Christo) is included here, posed several 
problems about flow in branched tubes and application to human arteries in which atherosclerosis has critical clinical impact. Our studies, of which a segment is described here, have utilized the computational and visualization framework already detailed by Sobel et al [9] to examine pulsatile flow in models of branched arteries and, in particular, to search for and examine particular features of interest such as conditions for flow separation opposite the entrance to a side branch, flow separation over the lip of a side branch, local instability, and alteration in secondary flow patterns in a curved tube near a side branch.

In conclusion, this paper illustrates how computational fluid dynamics together with computer visualization form a useful complement to physical experiments and, when broader knowledge of fluid dynamics is also applied, help to make orderly and systematic interpretation of complex phenomena.

\section{Acknowledgements}

This work was supported in part by NSF (CNS-0427374, CCR-0086065).

\section{References}

1. C. Caro, J.M. Fitz-Gerald, and R.C. Schroter. Arterial wall shear and distribution of early atheroma in man. Nature, 223:1159-1161, 1969.

2. C. Caro, J.M. Fitz-Gerald, and R.C. Schroter. Atheroma and wall shear. Observations, correlations, and a proposal for a shear-dependent mass transfer mechanism for atherogenesis. Proc. Roy. Soc. B, 177:109-159, 1971.

3. N. Woolf. Pathology of atherosclerosis. Butterworth Scientific, London, 1982.

4. M. J. Davies. Atlas of coronary artery disease. Lippincott-Raven, Philadelphia PA, 1998.

5. J.L. Christo and P.D. Richardson. Flow in a tube with a side branch. In Proc 11th Annual North East Bioengineering Conf., pages 139-142, 1985.

6. P.D. Richardson and J. Christo. Flow separation opposite a side branch. In D. Liepsch, editor, Biofluid Mechanics: Blood Flow in Large Vessels, 1990.

7. W.R. Dean. The streamline motion of a fluid in a curved pipe. Phil. Mag., (7th Ser) 4:208-233, 1927.

8. K. Tanishita, K. Nakano, P.D. Richardson, P.M. Galletti, M. Sugwara, and Y. Sakurai. Augmentation of gas transfer with pulsatile flow in the coiled tube membrane oxygenator design. Trans. Amer. Soc. Artif. Organs, 26:561-565, 1980.

9. J.S. Sobel, A.S. Forsberg, D.H. Laidlaw, R.C. Zeleznik, D.F. Keefe, I. Pivkin, G.E. Karniadakis, P. Richardson, and S. Swartz. Particle flurries - synoptic 3d pulsatile flow visualization. IEEE Comp. Graph. Appl., 24(2):76-85, 2004. 